\title{
Vorwort zu Band II meiner Gesammelten Werke in 5 Bänden
}

\section{Die Herrschaft über den Grund des Erfolges als Kriterium der Gleichstellung von aktivem Tun und (unechtem) Unterlassen}

1. Für die Struktur und Methoden der Rechtsfindung im Rechtsstaat, die ich in Band 1 meiner Gesammelten Werke entwickelt und zuletzt immer stärker auf das Strafrecht hin konzentriert habe, ${ }^{1}$ stellt der hiermit vorgelegte Band 2 gewissermaßen die Probe aufs Exempel dar. Er betrifft das materielle Strafrecht (während das Strafverfahrensrecht den Gegenstand von Band 3 bilden wird) und hier wiederum ein kompaktes Thema des Allgemeinen Teils, nämlich die Täterschaft. An diesem Thema arbeite ich seit einem halben Jahrhundert, beginnend mit meiner 1970 abgeschlossenen und 1971 publizierten Dissertation über „Grund und Grenzen der unechten Unterlassungsdelikte“ und seitdem immer wieder auf den Prüfstand gestellt und weiter ausgebaut. Weil die damalige Monographie seit langem vergriffen ist, international aber immer noch eine Rolle spielt $^{2}$ und gewissermaßen den Referenzpunkt für die weiteren Arbeiten bildet, wird sie hier als unveränderte Neuauflage vorgelegt. Hinsichtlich der weiteren Entwicklung war ich aus Raumgründen zu einer strengen Auswahl gezwungen und muss deshalb für viele einzelne Fragen auf die dazu publizierten speziellen Abhandlungen verweisen. ${ }^{3}$

2. Wenn ich heute mit einem Abstand von 5 Jahrzehnten meine ursprüngliche Konzeption der unechten Unterlassungsdelikte, die den 1. Teil dieses Bandes bildet und im 2. und 3. Teil durch eine Auseinandersetzung mit Missverständnissen und dem herrschenden Eklektizismus weitergeführt wird, vor dem Hintergrund meiner heutigen allgemeinen Tätertheorie sehe, die nachfolgend in Teil 4-6 beschrieben wird, so käme ich fast in Versuchung, mich für einen guten Menschen zu halten, weil ich mir damals in meinem dunklen Drange des rechten Weges offenbar wohl bewusst ${ }^{4}$ gewesen bin:

Weil das aktive Tun und die begehungsgleiche Unterlassung aus demselben Straftatbestand bestraft werden, muss sich zwischen ihnen eine den Grund der

1 Rechtsfindung im Rechtsstaat und Rechtsdogmatik als ihr Fundament, 2020.

2 Nachw. u. Fn. 16.

3 Ein Nachweis meiner weiteren Beiträge zur Täterlehre, die in diesen Band nicht aufgenommen werden konnten, findet sich $\mathrm{u}$. in Fn. 20.

4 Goethe, Faust I, Prolog im Himmel, Vers 328. 
Bestrafbarkeit betreffende Gemeinsamkeit aufweisen lassen bzw., anders formuliert, können nur solche Unterlassungen ebenso wie aktive Handlungen und damit als „unechte“ bestraft werden, wenn sie unter dem für die Strafdrohung maßgeblichen normativen Aspekt mit diesen vergleichbar sind. Es war deshalb nur konsequent, dass unter der Herrschaft des Kausalmonismus nach einer Vergleichbarkeit in der Kausalität gesucht wurde, indem zunächst durch die Ingerenztheorie ${ }^{5}$ auf die Kausalität der Vorhandlung abgestellt wurde, was aber auf eine Verletzung des Schuldprinzips vermöge der Bestrafung des bloßen dolus subsequens hinauslief. Um diesem Vorwurf zu entgehen, wurde in der Interferenztheorie ${ }^{6}$ die Niederkämpfung des Rettungsimpulses in der Psyche des Täters als Ursache präsentiert, aber dadurch mit allen Regeln der Kunst gebrochen. Denn die Bestrafung innerpsychischer Vorgänge ist nur in einem totalitären Gottesstaat (oder vielleicht in einem zukünftigen Überwachungsstaat?) vorstellbar, zumal deren forensischer Nachweis außerhalb von Folter so gut wie ausgeschlossen war. Und selbst wenn die Hirnforschung in Zukunft einmal das Gedankenlesen ermöglichen (und ein zukünftiger Überwachungsstaat es praktizieren?) würde, ${ }^{7}$ bliebe die Interferenztheorie immer noch grotesk falsch, weil danach ein völlig Unbeteiligter, der aufgrund einer supererogatorischen Moral zunächst an Hilfe gedacht und dann doch darauf verzichtet hätte, wie ein Begehungstäter bestraft werden müsste, während ein völlig hartgesottener Garant, dem eine Hilfeleistung nicht einmal in den Sinn gekommen war, straflos ausgehen müsste.

Mit der Ablösung des Kausalmonismus durch den Neukantianismus und dessen Forderung nach einer wertbeziehenden, d.h. normativistischen Denkweise kam es in der Unterlassungsdogmatik kurioserweise ebenfalls $\mathrm{zu}$ einem Doppelfehler, indem die Gleichstellbarkeit des Unterlassens mit aktivem Tun einerseits als eine Frage der Rechtswidrigkeit angesehen und die Lösung in der Akzessorietät des Strafrechts zu allen anderen Rechtsgebieten gesucht wurde, während andererseits (vor allem in der Rechtsprechung) die aus dem Kausalmonismus stammende und damit eigentlich obsolete Ingerenztheorie beibehalten wurde. Die Anknüpfung an eine außerstrafrechtliche (und in diesem Sinne „formelle“) Rechtspflicht aus Gesetz oder Vertrag eröffnete jedoch keine neuen Perspektiven, sondern bedeutete einen Rückschritt zu der 100 Jahre zuvor von Feuerbach skizzierten Konzeption, die sich auf der Suche nach dem strafrechtli-

5 Krug, Hitzig‘s Annalen der deutschen und ausländischen Criminal-Rechts-Pflege Bd. 63 (1853), 201 ff.; Glaser, Abhandlungen aus dem österreichischen Strafrecht, Wien 1858, $289 \mathrm{ff}$.

6 v. Buri GS 21, 129 f.; ders. GS 27, 26 ff.; ders., Über Causalität und deren Verantwortung, 1872, S. $15 \mathrm{ff}$.

$7 \mathrm{Zu}$ solchen evtl. Möglichkeiten Merkel, ZStW 121 (2009), 919 ff. 
chen Gleichstellungsgrund an dem öfters, aber nicht notwendig damit verbundenen Epi-Phänomen der zivilrechtlichen Pflicht verausgabte, wie durch die beiden Kindermädchenfälle manifestiert wird und heute nahezu unstreitig ist: Während ein Kindermädchen, das das von ihr beaufsichtigte Kleinkind bei einem Spaziergang von einem schlecht gelaunten Dackel beißen lässt, auch dann wegen Körperverletzung durch Unterlassen strafbar ist, wenn sich ihr zivilrechtlicher Arbeitsvertrag als nichtig herausstellt, bleibt der gültige Vertrag ohne strafrechtliche Bedeutung, wenn die Arbeitsstelle tatsächlich nicht angetreten und die Betreuung des Kindes gar nicht übernommen wird. Dieser Irrweg einer Anknüpfung an bloße Epi-Phänomene traf auch die (innerlich mit der formellen Rechtspflichttheorie niemals verbundene) Ingerenztheorie, die vom Bundesgerichtshof zunächst auf pflichtwidrige Vorhandlungen reduziert, dann aber im Fall der Produkthaftung wieder auf jede Vorhandlung ausgedehnt und sogar noch (was in einem Kausalansatz diesen selbst kompromittiert) für übertragbar erklärt wurde. ${ }^{8}$

3. Dass die Ersetzung des Kausalmonismus durch die außerstrafrechtlichen Rechtspflichten von der spezifisch strafrechtlichen Gleichstellbarkeitsprüfung wegführt, erkannten bereits Schaffstein und Nagler ${ }^{9}$, wobei letzterer für die strafrechtsspezifische Gleichstellung den Ausdruck „Garantenstellung“ prägte, doch vermochten sie zu einer besseren Lösung im Banne des nationalsozialistischen Strafrechtsdenkens nicht vorzudringen. Den Schlüssel hierzu hielt dann erst Armin Kaufmann in der Hand, als er die Zweiteilung der anerkannten Garantenstellungen in solche zur Überwachung einer bestimmten Gefahrenquelle oder zum Schutz eines bestimmten Rechtsgutsobjekts registrierte. ${ }^{10}$ Aber anstatt anschließend in den Fußstapfen seines Lehrers Hans Welzel nach den diesen beiden Gruppen zugrundeliegenden sachlogischen Strukturen zu fragen, postulierte er zwischen Begehung und Unterlassung das sog. Umkehrprinzip, ${ }^{11}$ aus dem per definitionem keine Gleichstellbarkeit, sondern nur Verschiedenheit abzuleiten gewesen wäre. Stattdessen muss die Gleichstellungsfrage genau umgekehrt gestellt werden: Lässt sich ein Prinzip benennen, unter das sowohl die Begehung durch aktives Tun als auch die unumstrittenen Fälle der unechten Unterlassung subsumiert werden können? Die Lösung dieser Aufgabe dämmerte mir, als mir die Ungenauigkeit der üblichen Redeweise aufging, dass das Strafrecht Handlungen bestrafe. In Wahrheit wird ja nicht die Handlung, sondern es wird ein Mensch wegen seiner Handlung bestraft, und zwar weil es

8 In der Lederspray-Entscheidung BGHSt 37, 106.

9 Schaffstein, FS Graf Gleispach, 1936, S. 70 ff.; Nagler GS 111, 1 ff.

10 Die Dogmatik der Unterlassungsdelikte, 1959, S. 283.

11 aaO. (Fn. 10), S. $87 \mathrm{ff}$. 
allein von seinem Willen, seiner Entscheidung abhängt, ob er diese Handlung begeht oder nicht. Den unverzichtbaren Nexus bildet deshalb die Herrschaft des Menschen über seinen Körper (über seine Handlungen), die etwa bei unwiderstehlichen Reflexen fehlen kann und dann auch jede strafrechtliche Verantwortlichkeit ausschließt. Über diese Handlung beherrscht er wiederum das zur Rechtsgutsverletzung führende Geschehen, das seinen für die Zurechnung entscheidenden Grund in der Handlung des Täters findet. Sobald man den durch Evidenzerlebnisse abgesicherten, festen Kern der unechten Unterlassungsdelikte in die Betrachtung einbezieht, zeigt sich eine naturgemäß nicht identische, aber durchaus vergleichbare Form der Geschehensbeherrschung: entweder durch die Beherrschung anderer wesentlicher Erfolgsursachen, die sogar in phänotypischer Weise mit dem Ablaufenlassen eines im Frühstadium willentlich beherrschbaren Reflexes verglichen werden kann; oder bei der zweiten Gruppe die Herrschaft über die spezifische Hilflosigkeit des Rechtsgutsobjekts, derzufolge dieses sich nicht ausreichend selbst verteidigen kann und sich in die Obhut eines Anderen begeben hat oder gegeben worden ist.

Bei dem von mir vor einem halben Jahrhundert hierfür geprägten Oberbegriff der „Herrschaft über den Grund des Erfolges“ handelt es sich deshalb geradezu um das Muster eines Typus-Begriffs, dessen rechtstheoretische Entschlüsselung ${ }^{12}$ an diesem Beispiel weiter vorangetrieben werden kann: Die mithilfe von Analogien vorzunehmende Konkretisierung setzt einen Gegenbegriff sowie einen festen Extensionskern mit einem Bestand an evidenten „Archetypen" (d.h. in der Rechtsfolge unbestrittener und semantisch durch die Benutzung klassifikatorischer Termini klar bestimmter Fallgruppen) voraus, ${ }^{13}$ bei den strafrechtlichen Garantenstellungen als Gegenbegriff den zufällig abwendungsmächtigen quivos ex populo und als Archetypen die Kindermädchen und die stillende Mutter in ihrer Obhutsherrschaft über das Kleinkind/den Säugling sowie den Hunde- oder Fahrzeugführer in ihrer Aufsichtsherrschaft über den gehorsamen Hund oder das Kraftfahrzeug. Durch Konkretisierung ,von oben“ und Abstrahierung ,von unten“ können dadurch die auf einer mittleren Abstraktionshöhe angesiedelten Garantenstellungstypen (in der Dogmatik traditionell als „Garantenstellungen“ bezeichnet) entwickelt werden; etwa in der 1. Gruppe die Gefahrengemeinschaft, die (ihre alltägliche Form darstellende) enge Lebensgemeinschaft und die Übernahme von Schutzfunktionen über relativ hilflo-

12 Dazu bereits Band I (Fn. 1), S. 168 ff., 312 f.; in diesem Band u. 5. Teil, S. 530 m. Fn. 42.

13 Die Analyse der hinreichenden quantitativen Gesamtausprägung der verschiedenen Züge des Typus (dazu Nachw. u. S. 530 in Fn. 42) wird also durch diese Heuristiken (zum „Gegenbegriff“ s. Schünemann, FS f. Otto, 2007, S. 777, $791 \mathrm{ff.,} \mathrm{zu} \mathrm{den} \mathrm{den} \mathrm{Extensionskern} \mathrm{bildenden}$ Archetypen u. 3. Teil, S. 474) unterstützt. 
se Personen wie diejenige des Arztes über seinen kranken Patienten oder diejenige des Markenwarenproduzenten zur Aufklärung des Konsumenten über die vermöge seiner Produktbeobachtung nachträglich entdeckten gefährlichen Produktmängel ${ }^{14}$; oder in der 2. Gruppe die Herrschaft über gefährliche Sachen oder Verrichtungen bis hin zur Geschäftsherrenhaftung. ${ }^{15}$

4. Eigenartigerweise hat die Gleichstellungstheorie der Herrschaft über den Grund des Erfolges zwar international eine bemerkenswerte Resonanz, ${ }^{16}$ innerhalb der deutschen Strafrechtsdogmatik aber - bis auf einzelne, freilich äußerst gewichtige Zustimmung ${ }^{17}$ - nur oberflächliche Beachtung und in diesem Rahmen Kritik erfahren. Nicht weniger eigenartig ist es, dass es bezüglich der Gleichstellungsfrage anders als in praktisch allen anderen strafrechtsdogmatischen Fragen des Allgemeinen Teils eigentlich keine herrschende Meinung gibt, sondern nur zahlreiche höchst unterschiedliche und disparate Garantentheorien im Schrifttum, während sich die Rechtsprechung von einem systematisch konsistenten Begründungskonzept seit langem verabschiedet hat und nur noch in ihrer eigenen Kasuistik lebt. Eine diesen Namen verdienende Strafrechtsdogmatik kann sich deshalb nicht damit zufriedengeben, dass ein großes gemeinsames Reservoir an Fallösungen existiert. Denn solange diese nicht in einer systematischen Theorie erfasst sind, handelt es sich um bloße Kadijustiz, die überdies dann nicht weiterhilft, wenn neue Konstellationen auftreten. Ein Beispiel bietet die Garantenstellung des nach amerikanischem Vorbild eingerichteten Compliancebeauftragten, die - vom Bundesgerichtshof in einem nicht mehr überbietbar oberflächlichen obiter dictum bejaht, im Schrifttum aber heillos umstritten $^{18}$ - im Lichte der Herrschaftstheorie ihren Grund wie ihre (engen)

14 Im Ansatz bereits Schünemann, in: Gimbernat/Schünemann/Wolter (Hrsg.), Internationale Dogmatik der objektiven Zurechnung und der Unterlassungsdelikte, 1995, S. 49, 70 f.; zur Einschränkung auf Markenware ders., in: 50 Jahre Bundesgerichtshof - Festgabe aus der Wissenschaft, 2000, S. 621, 640 f.; u. 2. Teil, S. 462 ff.; zust. Schmucker, Die „Dogmatik“ einer strafrechtlichen Produktverantwortung, 2001, S. 150 f. In der Grundkonstruktion ebenfalls zust., aber ohne die Einschränkung auf Markenware Roxin, Strafrecht AT II, 2006, S. 783.

15 Dazu eingehend bereits Schünemann, Unternehmenskriminalität und Strafrecht, 1979, nunmehr in Band 5 Teil 1.

16 Eingehende Nachw. u. im 2. Teil, S. 465, 468. Ferner ist meine Monographie von $1971 \mathrm{im}$ Jahr 2009 in spanischer Übersetzung von Cuello Contreras und Serrano González de Murillo bei Marcial Pons in Madrid-Barcelona-Buenos Aires erschienen („Fundamento y Límites de los Delitos de Omisión Impropia“).

17 Vor allem bei Roxin (Fn. 14), S. 717 ff. S: ferner Schmucker (Fn. 14) sowie Berster, Die völkerstrafrechtliche Unterlassungsverantwortlichkeit, 2008; ders., Das unechte Unterlassungsdelikt: Der gordische Knoten des Allgemeinen Teils, 2014, und dazu u. S. 477 Fn. 20.

18 BGHSt 54, 44, 49 und zur Kontroverse statt aller Knauer u. Rotsch, FS f. Imme Roxin, 2012, S. 465, 485. 
Grenzen in der sektoralen Informationsherrschaft findet, die der Compliancebeauftragte als „innerbetriebliches Auge“ des Vorstandes erlangt, weshalb eine von ihm unterlassene Information des Entscheidungsorgans einer aktiven Täuschung gleichsteht. ${ }^{19}$ Bei der Entwicklung der Herrschaftstheorie und der Kritik anderer Gleichstellungstheorien geht es also auch heute noch nicht um Dogmengeschichte, sondern um die Auffindung des aktuell geltenden Rechts, für die der Wiederabdruck meiner seit Jahrzehnten vergriffenen Monographie als Teil I dieses Bandes das Fundament zu legen beansprucht, das die heutigen Kritiker schlimmstenfalls als schief und brüchig, nicht aber als obsolet verurteilen könnten. Umgekehrt beweisen der in Teil 2 und Teil 3 dieses Bandes zu findende Weiterbau des Fundaments wie auch der Ausbau des Gesamtgebäudes in Band V (in Gestalt der Geschäftsherrenhaftung) und in weiteren, an anderer Stelle publizierten Abhandlungen ${ }^{20}$ die Tragfähigkeit und systematische Ver-

19 Es handelt sich deshalb, wenn der Compliancebeauftragte wie üblich keine innerbetriebliche Anweisungsmacht hat, um eine Herrschaft über die auf Unkenntnis beruhende Hilflosigkeit, nicht über eine Gefahrenquelle, weshalb das Strafrecht nur die Aufklärung des Weisungsbefugten über die Gefahr, nicht aber beispielsweise die Einschaltung der Polizei verlangt eine aus der Natur der Garantenstellung folgende Begrenzung, für die dem BGH bei seinen kursorischen Bemerkungen von vornherein das dogmatische Sensorium fehlte.

20 Unternehmenskriminalität und Strafrecht - Eine Untersuchung der Haftung der Wirtschaftsunternehmen und ihrer Führungskräfte nach geltendem und geplantem Straf- und Ordnungswidrigkeitenrecht, 1. Aufl. 1979, 2. Aufl. in Band V der Gesammelten Werke; Kommentierung von §25-31 StGB („Täterschaft und Teilnahme“), in: Laufhütte/Rissing-van Saan/ Tiedemann (Hrsg.), Leipziger Kommentar zum StGB, Berlin 12. Aufl. 2007, Band I S. 1813 ff.; Kommentierung von $\S 14$ StGB („Handeln für einen anderen“), in: Cirener/Radtke/Rissing-van Saan/Rönnau/Schluckebier (Hrsg.), Leipziger Kommentar zum StGB, Berlin 13. Aufl. 2020, Band I S. $939 \mathrm{ff}$.

Zur Kritik der Ingerenz-Garantenstellung, GA 1974, 231 ff.; Besondere persönliche Verhältnisse und Vertreterhaftung im Strafrecht, in: Zeitschrift für Schweizer Recht 1978 I, S. 131 ff.; Die Bedeutung der „Besonderen persönlichen Merkmale“ für die strafrechtliche Teilnehmerund Vertreterhaftung, Jura 1980, S. 354 ff., 568 ff.; Strafrechtsdogmatische und kriminalpolitische Grundfragen der Unternehmenskriminalität, wistra 1982, S. 41 ff.; Die Unterlassungsdelikte und die strafrechtliche Verantwortlichkeit für Unterlassungen, ZStW 96 (1984), 287 ff.; Die deutschsprachige Strafrechtswissenschaft nach der Strafrechtsreform im Spiegel des Leipziger Kommentars und des Wiener Kommentars, 1. Teil: Tatbestands- und Unrechtslehre, GA 1985, 341 ff.; Die strafrechtliche Verantwortung der Unternehmensleitung im Bereich von Umweltschutz und technischer Sicherheit, in: Breuer/Kloepfer/Marburger/Schröder (Hrsg.), Umweltschutz und technische Sicherheit im Unternehmen, 1994, S. 137 ff.; Die Strafbarkeit der juristischen Person aus deutscher und europäischer Sicht, in: Schünemann/Suarez Gonzalez (Hrsg.), Bausteine des europäischen Wirtschaftsstrafrechts, 1994, S. 265 ff.; Zum gegenwärtigen Stand der Dogmatik der Unterlassungsdelikte in Deutschland, in: Gimbernat/Schünemann/Wolter (Hrsg.), Internationale Dogmatik der objektiven Zurechnung und der Unterlassungsdelikte. 1995, S. 49 ff.; Begründung und Entwurf eines Gesetzes zur Bekämpfung der Unternehmens- 
vollkommnung von Fundament und Gebäude - bis hin zum fugenlosen Einbau der erwähnten Garantenstellung des Compliancebeauftragten, der, um im Bilde zu bleiben, im Herrschaftsgebäude von jedem Polier geleistet werden kann, während im Wirrwarr der disparaten Garantenstellungen die prominentesten Architekten daran verzweifeln.

\section{Das Herrschaftsprinzip als allgemeine Struktur der Täterschaft}

Damit ist aber die Leistungsfähigkeit des Herrschaftsprinzips als über die Lösung der Gleichstellungsfrage entscheidendem tertium comparationis längst noch nicht erschöpft. Vielmehr haben meine späteren Studien gezeigt, dass es zugleich den Ansatzpunkt für die Auflösung zahlreicher anderer gordischer Knoten in der Dogmatik der strafrechtlichen Beteiligungslehre liefert.

1. Das betrifft zum ersten die sog. strafrechtliche Vertreterhaftung, die vor allem für die Täterschaftsfrage im Wirtschaftsstrafrecht eine zentrale Rolle spielt, lange Zeit aber nur wie ein rätselhafter zivilistischer Fremdkörper im Strafrecht aufgefasst und mit formalen Pflichttheorien bearbeitet wurde, die mit den materiellen Zurechnungskategorien des rechtsgüterschützenden Strafrechts wenig zu tun hatten. Indem der Gesetzgeber ein und denselben Terminus der „besonderen persönlichen Merkmale“ sowohl für Zurechnungsfragen bei der

kriminalität, in: Schünemann (Hrsg.), Unternehmenskriminalität; Deutsche Wiedervereinigung Band III, 1996, S. 153 ff.; Unternehmenskriminalität, in: Canaris/Heldrich/Hopt/Roxin/ K. Schmidt/Widmaier (Hrsg.), 50 Jahre Bundesgerichtshof - Festgabe aus der Wissenschaft, 2000, Band IV, S. 621 ff.; Zur Regelung der unechten Unterlassung in den Europa-Delikten, in: Tiedemann (Hrsg.), Wirtschaftsstrafrecht in der Europäischen Union, 2002, S. 103 ff.; Die kriminalpolitischen und dogmatischen Grundfragen der Unternehmenskriminalität, in: FS für Hans-Joachim Rudolphi, 2004, S. 295 ff.; Brennpunkte des Strafrechts in der entwickelten Industriegesellschaft - Reflexionen zu den Beiträgen des Symposiums -, in: Hefendehl (Hrsg.), Empirische und dogmatische Fundamente, kriminalpolitischer Impetus. Symposium für Bernd Schünemann zum 60. Geburtstag, 2005, V. Die stürmische Ausdehnung der mittelbaren Täterschaft, S. 349 ff.; Die Rechtsfigur des „Täters hinter dem Täter“ und das Prinzip der Tatherrschaftsstufen, in: Festschrift für Friedrich-Christian Schroeder, 2006, S. 399 ff.; Vom kriminalpolitischen Nutzen und Nachteil eigenhändiger Delikte, in: Festschrift für Heike Jung, 2007, S. 881 ff.; Die „besonderen persönlichen Merkmale“ des § 28 StGB, in: Festschrift für Wilfried Küper, 2007, S. 561 ff.; Was heißt und zu welchem Ende betreibt man Strafrechtsdogmatik? Zu Fischers These der „fremden seltsamen Welten“ anhand aktueller BGH-Urteile zu Begriff und Funktion der „besonderen persönlichen Merkmale“ im Strafrecht, GA 2011, 445 ff.; Die Rechtsfigur der sog. Einheitstäterschaft im Strafrecht - Kritik eines dogmatischen Monstrums, GA 2020, $224 \mathrm{ff}$. 
Strafzumessung in $\S 28$ StGB als auch für die Täterqualifikation bei Sonderdelikten in $\S 14$ StGB verwendet hat, ist diese Konfusion auf den ersten Blick zu einem Knoten geschürzt worden, „dessen Lösung jeden Menschenwitz (zu) übersteigen“ scheint. Aber so wie die Abgrenzung von Tat- und Rechtsfrage, der dieses geflügelte Wort von Graf zu Dohna einmal galt, ${ }^{21}$ durch die sprachanalytische Unterscheidung von Objektsprache und Metasprache mittlerweile gelungen ist, wie in Band 3 der Gesammelten Werke näher ausgeführt werden wird, lässt sich die Struktur der sog. Vertreterhaftung bei Sonderdelikten mit „,besonderen persönlichen Merkmalen“ unschwer entschlüsseln, weil es dabei um nichts anderes geht als um „eine Form der Übernahme einer Garantenstellung“, 22 dergestalt dass der im Straftatbestand durch eine Statusbezeichnung beschriebene Sonderdeliktstäter, der „intraneus“, seine besondere materielle (Garanten-)Stellung zum rechtsgutverletzenden Geschehen (nämlich seine Herrschaft entweder über eine Gefahrenquelle oder über die Hilflosigkeit des Rechtsguts) auf einen anderen überträgt, der dadurch selbst in die die Täterqualifikation begründende Herrschaftsbeziehung einrückt - und nicht etwa nur in eine formale „Pflichtigkeit“, die ja mit dem tatbestandlichen Gebot oder Verbot identisch ist und deshalb einen reinen Zirkelschluss bedeutet.

2. Die durch die Entschlüsselung der Vertreterhaftung gewonnene Erkenntnis, dass es sich bei den hierfür in Betracht kommenden und damit den meisten Sonderdelikten um Garantensonderdelikte handelt, macht zugleich den Weg frei für eine adäquate Analyse des Unrechts der verbleibenden (seltenen) „reinen Pflichtdelikte“ und der durch die moderne Strafrechtsdogmatik wie Zombies geisternden eigenhändigen Delikte.

a) Reine Pflichtdelikte kann es in einem liberalen Rechtsstaat, in dem der Overkill des Strafrechts allein als Ultima Ratio zum Rechtsgüterschutz eingesetzt werden darf, nur in einem engen Rahmen geben. Als Rechtsgut kommen bei ihnen staatliche oder gesellschaftliche Institutionen wie die Ehe oder das Berufsbeamtentum in Frage, Begehungsform ist die Verletzung institutioneller Pflichten. Diese kann in der Regel aber durch Sanktionen innerhalb der Institution ausreichend geahndet werden (vor allem wenn ausschließlich institutsinterne Pflichten verletzt werden), beispielsweise durch ein Scheidungsrecht des Partners bei ehewidrigem Verhalten oder eine Disziplinarstrafe im Beamtenrecht. Während deshalb die Bestrafung des sog. Ehebruchs in einem rechtsstaatlichen Strafrecht keinen legitimen Platz hatte und (viel zu spät ${ }^{23}$ ) abgeschafft worden ist, steht eine dem spezifischen Unrecht adäquate Abgrenzung

21 In: Das Strafprozeßrecht, 3. Aufl. 1929, S. 199.

22 So erstmals Schünemann, Ztschr. f. Schweizer Recht 1978, 155.

23 Nämlich erst durch das 1. StrRG vom 30.6. 1969 (BGBl I S. 645). 
der Bestrafung von der bloßen disziplinarrechtlichen Ahndung im Falle von Vorteilsannahmen durch Amtsträger bis heute aus und wird vom Gesetzgeber sogar konterkariert. ${ }^{24}$

b) Eine weiterführende Analyse der in einem modernen rechtsgüterschützenden Strafrecht ominösen Deliktsgruppe der eigenhändigen Delikte findet sich erstmals in Roxins Unterscheidung ${ }^{25}$ zwischen Tatbeständen, die eine sich aus vielen Einzelheiten aufbauende asoziale Persönlichkeitshaltung bestrafen wie Landstreicherei und Zuhälterei, ${ }^{26}$ solchen, die ein Verhalten allein wegen seiner vom Gesetzgeber bejahten Unsittlichkeit bestrafen wie Beischlaf zwischen Verwandten, Unzucht zwischen Männern und Sodomie, ${ }^{27}$ und den von Roxin sog. höchstpersönlichen Pflichtdelikten wie Meineid und Unfallflucht. ${ }^{28}$ Dass die ersten beiden Gruppen in einem rechtsstaatlichen Strafrecht keinen Platz haben, sollte eigentlich klar sein, auch wenn die Entkriminalisierung in der zweiten Gruppe bis heute nur dort vorangekommen ist, wo eine hinreichend starke Interessengruppe dahinter stand. Strafrechtsdogmatisch interessanter ist die dritte Gruppe der (phänotypisch) eigenhändigen Delikte, bei der es sich im Lichte der Herrschaftstheorie um „Vorfeldbestrafung von Garantensonderdelikten“ handelt, die unter 5 Voraussetzungen legitim ist: Der Täter muss eine individuelle Schlüsselposition für das geschützte Rechtsgut innehaben, die Tathandlung ist von hoher Plakativität, erfasst die gefährlichen Handlungen mit hoher Treffsicherheit unter Schonung berechtigter Interessen und kommuniziert eine individuelle Missachtung des Rechtsguts (von mir sog. persönliche Expressivität). ${ }^{29}$

3. $\mathrm{Zu}$ guter Letzt ermöglicht die vertiefte und umfassende Entfaltung des Herrschaftsprinzips auch eine überzeugende Entscheidung der neuerdings wieder entfesselten Streitfragen über die Reichweite und Konsequenzen des Tatherrschaftsprinzips bei den Begehungs-Gemeindelikten: Die methodologischen Regeln über die Konkretisierung eines Typusbegriffs und das Modell der Tatherrschaftsstufen liefern eine überzeugende Abgrenzung der mittelbaren Täterschaft für die im Zentrum der Diskussion stehende Fallgruppe der Organisationsherrschaft und beweisen dadurch zugleich, dass entgegen neuerdings

24 Dazu näher Schünemann, FS f. Otto (Fn. 13); ders., ZRP 2015, 68 ff.; ders., in: Hoven/Kubiciel (Hrsg.), Das Verbot der Auslandsbestechung, 2016, S. $25 \mathrm{ff}$.

25 Täterschaft und Tatherrschaft, 1. Aufl. 1963, S. 399 ff.

$26 \S \S 181$ a. F., 361 Nr. 3 a. F. StGB.

$27 \S \S 173,175$ a. F., 175b a. F. StGB (beachte heute $\S 3$ S. 1 Nr. 13 i. V. m. $\S 18$ Abs. 1 Nr. 4 Tierschutzgesetz - Ordnungswidrigkeit mit Androhung von Bußgeld bis zu $25.000 €$ ).

$28 \S \S 142,154$ StGB.

29 Dazu eingehend Schünemann, FS f. Jung, 2007, S. 881 ff. 
wieder zu hörenden Stimmen nicht der mindeste Anlass besteht, auf eine differenzierte Täterschaftsdogmatik überhaupt zu verzichten und sich stattdessen in die Sackgasse des Einheitstäterbegriffs zu flüchten. ${ }^{30}$

\section{Konsequenzen für das sog. Verbandsstrafrecht}

Durch diese Analyse der sachlogischen Strukturen der Täterschaft in einem rechtsgüterschützenden Strafrecht steht auch außer Frage, dass es kein eigentliches Strafrecht gegen Kollektive geben kann und dass deshalb auf die kriminalpolitischen Probleme der aus einem Kollektiv heraus verursachten Rechtsgüterverletzungen („Unternehmenskriminalität“) anders als durch eine simple Kopie der Geldstrafe reagiert werden muss. In der Erörterung dieser Frage wird ein Schwerpunkt des Bandes V meiner Gesammelten Werke liegen, doch gehe ich auf einige Grundlagen bereits im Rahmen der allgemeinen Tätertheorie ein (unten Vierter Teil Abschnitt II, S. 490 ff.) und habe diese Passage deshalb auf den heutigen Diskussionsstand aktualisiert.

30 Dazu näher u. 5. Teil, S. 521 ff., sowie Schünemann, Die Rechtsfigur der sog. Einheitstäterschaft im Strafrecht - Kritik eines dogmatischen Monstrums, GA 2020, $224 \mathrm{ff}$. 